\title{
A new characterization of Mathieu-groups by the order and one irreducible character degree
}

Haijing $X u^{1}$, Yanxiong Yan ${ }^{1,2}$ and Guiyun Chen ${ }^{1 *}$

\section{"Correspondence:}

gychen@swu.edu.cn

'School of Mathematics and

Statistics, Southwest University,

Chongqing, 400715, China

Full list of author information is

available at the end of the article

\begin{abstract}
The main aim of this article is to characterize the finite simple groups by less character quantity. In fact, we show that each Mathieu-group $G$ can be determined by their largest and second largest irreducible character degrees.

MSC: $20 C 15$
\end{abstract}

Keywords: finite group; simple group; character degree

\section{Introduction and preliminary results}

Classifying finite groups by the properties of their characters is an interesting problem in group theory. In 2000, Huppert conjectured that each finite non-abelian simple group $G$ is characterized by the set $c d(G)$ of degrees of its complex irreducible characters. In [1-4], it was shown that many non-abelian simple groups such as $L_{2}(q)$ and $S_{z}(q)$ satisfy the conjecture. In this paper, we manage to characterize the finite simple groups by less character quantity. Let $G$ be a finite group; $L(G)$ denotes the largest irreducible character degree of $G$ and $S(G)$ denotes the second largest irreducible character degree of $G$. We characterize the five Mathieu groups $G$ by the order of $G$ and its largest and second largest irreducible character degrees. Our main results are the following theorems.

Theorem A Let $G$ be a finite group and let $M$ be one of the following Mathieu groups: $M_{11}$, $M_{12}$ and $M_{23}$. Then $G \cong M$ if and only if the following conditions are fulfilled:

(1) $|G|=|M|$;

(2) $L(G)=L(M)$.

Theorem B Let $G$ be a finite group. Then $G \cong M_{24}$ if and only if $|G|=\left|M_{24}\right|$ and $S(G)=$ $S\left(M_{24}\right)$.

Theorem C Let $G$ be a finite group. If $|G|=\left|M_{22}\right|$ and $L(G)=L\left(M_{22}\right)$, then either $G$ is isomorphic to $M_{22}$ or $H \times M_{11}$, where $H$ is a Frobenius group with an elementary kernel of order 8 and a cyclic complement of order 7 .

We need the following lemmas.

\section{Springer}

(0) 2013 Xu et al.; licensee Springer. This is an Open Access article distributed under the terms of the Creative Commons Attribution License (http://creativecommons.org/licenses/by/2.0), which permits unrestricted use, distribution, and reproduction in any medium, provided the original work is properly cited. 
Lemma 1 Let $G$ be a non-solvable group. Then $G$ has a normal series $1 \unlhd H \unlhd K \unlhd G$ such that $K / H$ is a direct product of isomorphic non-abelian simple groups and $|G / K| \mid$ $|\operatorname{Out}(K / H)|$.

Proof Let $G$ be a non-solvable group. Then $G$ has a chief factor $M / N$ such that $M / N$ is a direct product of isomorphic non-abelian simple groups. Hence $C_{G / N}(M / N) \cap M / N=$ $Z(M / N)=1$, and so

$$
M / N \cong \frac{C_{G / N}(M / N) \times M / N}{C_{G / N}(M / N)} \leq \frac{G / N}{C_{G / N}(M / N)} \lesssim \operatorname{Aut}(M / N) .
$$

Let $K / N=C_{G / N}(M / N) \times M / N$ and $H / N=C_{G / N}(M / N)$. Then $G / K \leq \operatorname{Out}(M / N)$ and $K / H \cong M / N$ is a direct product of isomorphic non-abelian simple groups. Thus $1 \unlhd H \unlhd$ $K \unlhd G$ is a normal series, as desired.

Lemma 2 Let $G$ be a finite solvable group of order $p_{1}^{a_{1}} p_{2}^{a_{2}} \cdots p_{n}^{a_{n}}$, where $p_{1}, p_{2}, \ldots, p_{n}$ are distinct primes. If $k p_{n}+1 \nmid p_{i}^{a_{i}}$ for each $i \leq n-1$ and $k>0$, then the Sylow $p_{n}$-subgroup is normal in $G$.

Proof Let $N$ be a minimal normal subgroup of $G$. Then $|N|=p^{m}$ for $G$ is solvable. If $p=p_{n}$, by induction on $G / N$, we see that normality of the Sylow $p_{n}$-subgroup in $G$. Now suppose that $p=p_{i}$ for some $i<n$. Now consider $G / N$. By induction, the Sylow $p_{n}$-subgroup $P / N$ of $G / N$ is normal in G/N. Thus $P \unlhd G$. Let $Q$ be a Sylow $p_{n}$-subgroup of $P$. Then $P=N Q$. By Sylow's theorem, $\left|P: N_{P}(Q)\right|=p_{i}^{l}\left(l \leq m \leq a_{i}\right)$ and $p_{n} \mid p_{i}^{l}-1$. But this means that $k p_{n}+1 \mid$ $p^{a_{i}}$, and then $k=0$ by assumption. Hence $Q \unlhd P$ and $Q \unlhd G$.

\section{Proof of theorems}

Proof of Theorem A We only need to prove the sufficiency. We divide the proof into three cases.

Case $1.1 M=M_{11}$

In this case, we have $|G|=2^{4} \cdot 3^{2} \cdot 5 \cdot 11$ and $L(G)=55$. We first show that $G$ is nonsolvable. Assume the contrary. By Lemma 2, we know that the Sylow 11-subgroup of $G$ is normal in $G$. Let $N$ be the 11-Sylow subgroup of $G$. Since $N$ is abelian, we have $\chi(1)|| G / N \mid$ for all $\chi \in \operatorname{Irr}(G)$. But $L(G)=55$ and $55 \nmid|G / N|$, a contradiction. Therefore, $G$ is nonsolvable.

Since $G$ is non-solvable, by Lemma 1 , we get that $G$ has a normal series $1 \unlhd H \unlhd K \unlhd G$ such that $K / H$ is a direct product of isomorphic non-abelian simple groups and $|G / K| \mid$ $|\operatorname{Out}(K / H)|$. As $|G|=2^{4} \cdot 3^{2} \cdot 5 \cdot 11$, we have $K / H \cong A_{5}, A_{6}, L_{2}(11)$ or $M_{11}$.

We first assume that $K / H \cong A_{5}$. Since $\left|\operatorname{Out}\left(A_{5}\right)\right|=2$, we have $|G / K| \mid 2$ and $|H|=2^{t}$. $3 \cdot 11$, where $t=1$ or 2 . Let $\chi \in \operatorname{Irr}(G)$ such that $\chi(1)=L(G)=55$ and $\theta \in \operatorname{Irr}(H)$ such that $\left[\chi_{H}, \theta\right] \neq 0$. Then $\theta(1)=11$ by the Clifford theorem (see Theorem 6.2 in [5]). On the other hand, since $|H|=2^{t} \cdot 3 \cdot 11$, we have $H$ is solvable. Let $N$ be a Sylow 11-subgroup of $H$. Then $N \unlhd H$ by Lemma 2 . Hence $\theta(1)|| H / N \mid=2^{t} \cdot 3$, a contradiction.

By the same reason as above, one has that $K / H \nsubseteq A_{6}$.

Suppose that $K / H \cong L_{2}(11)$. Since $\left|\operatorname{Out}\left(L_{2}(11)\right)\right|=2$, we have $|G / K| \mid 2$ and so $|H|=2^{a} \cdot 3$, where $a=1$ or 2 . Let $\theta \in \operatorname{Irr}(H)$ such that $e=\left[\chi_{H}, \theta\right] \neq 0$ and let $t=\left|G: I_{G}(\theta)\right|$. Then $\theta(1)=1$ 
and $e t=\chi(1) / \theta(1)=55$. Since $|H|=2^{t} \cdot 3$, where $a=1$ or 2 , we have that $55 \nmid\left|\operatorname{Aut}\left(H / H^{\prime}\right)\right|$. Hence $t=1, e=55$. But $(55)^{2}=e^{2} t=\left[\chi_{H}, \chi_{H}\right]>|G: H|=2^{b} \cdot 3 \cdot 5 \cdot 11$, where $2 \leq b \leq 3$, a contradiction.

If $K / H \cong M_{11}$, by comparing the orders of $G$ and $M_{11}$, we have $|H|=1$. Therefore $G=$ $K \cong M_{11}$.

Case $1.2 M=M_{23}$

In this case, we have $|G|=2^{7} \cdot 3^{2} \cdot 5 \cdot 7 \cdot 11 \cdot 23$ and $L(G)=2^{3} \cdot 11 \cdot 23$. Then $O_{23}(G)=1$. If not, then $\left|O_{23}(G)\right|=23$ and $O_{23}(G)$ is abelian. Hence $L(G)=2^{3} \cdot 11 \cdot 23|| G / O_{23}(G) \mid$, a contradiction.

If $G$ is solvable, then the Sylow 23-subgroup of $G$ is normal in $G$ by Lemma 2, which leads to a contradiction as above. Therefore $G$ is non-solvable.

Since $G$ is non-solvable, by Lemma 1 , we get that $G$ has a normal series $1 \unlhd H \unlhd K \unlhd G$ such that $K / H$ is a direct product of isomorphic non-abelian simple groups and $|G / K| \mid$ $|\operatorname{Out}(K / H)|$. As $|G|=2^{7} \cdot 3^{2} \cdot 5 \cdot 7 \cdot 11 \cdot 23$, we have that $K / H$ can be isomorphic to one of the simple groups: $A_{5}, L_{2}(7), A_{6}, L_{2}(8), L_{2}(11), A_{7}, M_{11}, L_{3}(4), A_{8}, M_{22}$ and $M_{23}$.

We first assume that $K / H \cong A_{5}$. Since $\left|\operatorname{Out}\left(A_{5}\right)\right|=2$, we have $|G / K| \mid 2$ and $|H|=2^{m}$. $3 \cdot 7 \cdot 11 \cdot 23$, where $m=4$ or 5 . Suppose that $H$ is non-solvable. By Lemma $1, H$ has a normal series $1 \unlhd A \unlhd B \unlhd H$ such that $B / A$ is a direct product of isomorphic non-abelian simple groups and $|H / B||| \operatorname{Out}(B / A) \mid$. Since $|H|=2^{m} \cdot 3 \cdot 7 \cdot 11 \cdot 23$, we have $B / A \cong L_{2}(7)$ and $|H / B| \mid 2$. Thus $|A|=2^{a} \cdot 11 \cdot 23$, where $0 \leq a \leq 2$. Let $N$ be a Sylow 23-subgroup of $A$. Then $N \unlhd A$ by Lemma 2 . Hence we get a subnormal series of $G, N$ char $A \unlhd B \unlhd H \unlhd K \unlhd G$, which implies that $N \unlhd G$. But $O_{23}(G)=1$, a contradiction. If $H$ is solvable, then the Sylow 23-subgroup of $H$ is normal in $H$ by Lemma 2, which leads to a contradiction as before.

By the same arguments as the proofs of $K / H \cong A_{5}$, we show that $K / H$ cannot be isomorphic to one of the simple groups: $A_{6}, L_{2}(7), L_{2}(8), L_{2}(11), A_{7}, M_{11}, L_{3}(4), A_{8}$ and $M_{22}$.

If $K / H \cong M_{23}$, since $|G|=2^{7} \cdot 3^{2} \cdot 5 \cdot 7 \cdot 11 \cdot 23$, we have that $|H|=1$ and $G=K \cong M_{23}$.

Case $1.3 M=M_{12}$

In this case, $|G|=2^{6} \cdot 3^{3} \cdot 5 \cdot 11$ and $L(G)=2^{4} \cdot 11$. Since $11 \mid L(G)$, by the same arguments as the proofs of Case 1.2 , we have that $O_{11}(G)=1$.

We will show that $G$ is non-solvable. If $G$ is solvable, then the Sylow 11-subgroup of $G$ is normal in $G$ by Lemma 2, a contradiction. Therefore, $G$ is non-solvable.

By Lemma 1 , we get that $G$ has a normal series $1 \unlhd H \unlhd K \unlhd G$ such that $K / H$ is a direct product of isomorphic non-abelian simple groups and $|G / K||| \operatorname{Out}(K / H) \mid$. As $|G|=$ $2^{6} \cdot 3^{3} \cdot 5 \cdot 11$, we have $K / H \cong A_{5}, A_{6}, L_{2}(11), M_{11}$ or $M_{12}$.

By the same arguments as the proofs of Case 1.2, we can prove that $K / H$ cannot be isomorphic to $A_{5}$ or $A_{6}$.

Assume that $K / H \cong L_{2}(11)$. Since $\left|\operatorname{Out}\left(L_{2}(11)\right)\right|=2$, we have $|G / K| \mid 2$ and $|H|=2^{a} \cdot 3$, where $a=3$ or 4 . Suppose that $|G / K|=1$. Then $|H|=2^{4} \cdot 3^{2}$. Let $\chi \in \operatorname{Irr}(G)$ such that $\chi(1)=L(G)=2^{4} \cdot 11$ and $\theta \in \operatorname{Irr}(H)$ such that $e=\left[\chi_{H}, \theta\right] \neq 0$. Then $\chi(1)=\operatorname{et} \theta(1)=176$, where $t=\left|G: I_{G}(\theta)\right|$. Since $\chi(1) / \theta(1)|| G / H \mid$, we have that $\theta(1)=4$ or 8 . If $\theta(1)=4$, then et $=44$. Since $|H|=2^{4} \cdot 3^{2}$, we have that $H$ has at most eight irreducible characters of degree 4 . Hence $t \leq 4$. We assert that $I_{G}(\theta)=G$. If not, then $I_{G}(\theta)<G$. Let $U$ containing $I_{G}(\theta)$ be a maximal subgroup of $G$. Then $1 \leq|G: U||| G: I_{G}(\theta) \mid=4$. By checking the maximal subgroups of $L_{2}(11)$ (see ATLAS table in [6]), it is easy to get a contradiction. Hence $I_{G}(\theta)=G$, and so $t=1$ and $e=44$. But $e^{2} \cdot t=\left[\chi_{H}, \chi_{H}\right]>|G: H|$, a contradiction. If $\theta(1)=8$, then $\left|O_{3}(H)\right|=9$ and $I_{G}(\theta)=G$. Since $H \unlhd G$, we have that $O_{3}(H) \unlhd G$. Let $\lambda \in$ 
$\operatorname{Irr}\left(O_{3}(H)\right)$ such that $\left[\theta_{O_{3}(H)}, \lambda\right] \neq 0$. Since $\theta(1)=8$, we have $4 \leq\left|H: I_{H}(\lambda)\right| \leq 8$. But $I_{G}(\theta)=$ $G$, which implies that $4 \leq\left|G: I_{G}(\lambda)\right|=\left|H: I_{H}(\lambda)\right| \leq 8$. Let $S=\bigcap_{g \in G} I_{G}(\lambda)^{g}$. Then $S \unlhd G$ and $G / S \lesssim S_{8}$. By the Jordan-Hölder theorem, $S$ has a normal series $1 \unlhd O_{3}(H) \unlhd C \unlhd D \unlhd S$ such that $D / C \cong L_{2}(11)$ and $\left|C / O_{3}(H)\right|=1,2$ or 4 . Let $\alpha \in \operatorname{Irr}(S)$ such that $\left[\chi_{S}, \alpha\right] \neq 0$. Since $\chi(1) / \alpha(1)|| G / S \mid$, we have that $22 \mid \alpha(1)$. Since $\lambda^{g}$ is invariant in $S$, for each $g \in G$ and $4 \leq\left|G: I_{G}(\lambda)\right|$, we have that each irreducible character is invariant in $S$ and $O_{3}(H) \leq Z(S)$. Therefore, the following conclusions hold:

(a) $S \cong L_{2}(11) \times O_{3}(H)$ if $\left|C / O_{3}(H)\right|=1$;

(b) $S \cong\left(2 \cdot L_{2}(11)\right) \times O_{3}(H)$ or $\left(Z_{2} \times L_{2}(11)\right) \times O_{3}(H)$ if $\left|C / O_{3}(H)\right|=2$.

By checking the character table of $2 \cdot L_{2}(11)$ and $L_{2}(11)$, we see that both conclusions (a) and (b) are not satisfied with the above conditions. Now, we suppose that $\left|C / O_{3}(H)\right|=4$. Then $44 \mid \alpha(1)$. Since $O_{3}(H) \leq Z(S)$, one has that $C \cong O_{3}(H) \times B$, where $B$ is a group of order 4. Let $\beta$ be an irreducible component of $\alpha_{C}$ and $t_{1}=\left|S: I_{S}(\beta)\right|$. Then $\beta(1)=1$ and $t_{1}|\alpha(1) / \beta(1)| 44$. Since the indexes of the maximal subgroups of $S$ containing $I_{S}(\beta)$ divide $t_{1}$ and $t_{1}|| \operatorname{Aut}(C) \mid$, we have that $t_{1}=1$. Hence $\left[\alpha_{C}, \alpha_{C}\right]>|S: C|=2^{2} \cdot 3 \cdot 5 \cdot 11$, a contradiction.

Similarly, we can show that $|G / K| \neq 2$.

Suppose that $K / H \cong M_{11}$. Since $\left|\operatorname{Out}\left(M_{11}\right)\right|=\operatorname{Mult}\left(M_{11}\right)=1$, we have $G \cong H \times M_{11}$, where $|H|=2^{2} \cdot 3$. By checking the character table of $M_{11}$, we see that $G$ has no irreducible character of degree $L(G)=2^{4} \cdot 11$, a contradiction.

If $K / H \cong M_{12}$, since $|G|=2^{6} \cdot 3^{3} \cdot 5 \cdot 11$, we conclude that $|H|=1$ and $G=K \cong M_{12}$, which completes the proof of Theorem A.

Proof of Theorem B We only need to prove the sufficiency.

In this case, we have $|G|=2^{10} \cdot 3^{3} \cdot 5 \cdot 7 \cdot 11 \cdot 23$ and $S(G)=2^{2} \cdot 3^{2} \cdot 7 \cdot 23$. Let $\chi \in \operatorname{Irr}(G)$ such that $\chi(1)=S(G)$. If $O_{23}(G) \neq 1$, then $\left|O_{23}(G)\right|=23$, which implies that $\chi(1)|| G: N \mid$, a contradiction. Hence $O_{23}(G)=1$.

We have to show that $G$ is non-solvable. Assume the contrary, by Lemma 2, we have that the Sylow 23-subgroup is normal in $G$, a contradiction. Therefore, $G$ is non-solvable.

Since $G$ is non-solvable, by Lemma 1 , one has that $G$ has a normal series $1 \unlhd H \unlhd K \unlhd G$ such that $K / H$ is a direct product of isomorphic non-abelian simple groups and $|G / K| \mid$ $|\operatorname{Out}(K / H)|$. As $|G|=2^{10} \cdot 3^{3} \cdot 5 \cdot 7 \cdot 11 \cdot 23$, then $K / H$ can be isomorphic to one of the following simple groups: $A_{5}, L_{2}(7), A_{6}, L_{2}(8), L_{2}(11), A_{7}, U_{3}(3), M_{11}, L_{3}(4), A_{8}, M_{12}, M_{22}$, $M_{23}$ and $M_{24}$.

We first assume that $K / H \cong A_{5}$. Since $\left|\operatorname{Out}\left(A_{5}\right)\right|=2$, we have $|G / K| \mid 2$ and $|H|=2^{t} \cdot 3^{2}$. $7 \cdot 11 \cdot 23$, where $t=7$ or 8 . Let $\theta \in \operatorname{Irr}(H)$ such that $\left[\chi_{H}, \theta\right] \neq 0$. Since $\chi(1) / \theta(1)|| G / H \mid$, it implies that $23 \mid \theta(1)$. If $H$ is solvable, then $O_{23}(H) \neq 1$ by Lemma 2, which implies that $O_{23}(H)=O_{23}(G) \neq 1$, a contradiction. Thus $H$ is non-solvable. Then there exists a normal series of $H: 1 \unlhd N \unlhd M \unlhd H$ such that $M / N$ is a direct product of isomorphic non-abelian simple groups and $|H / M||| \operatorname{Out}(M / N) \mid$. As $|H|=2^{t} \cdot 3^{2} \cdot 7 \cdot 11 \cdot 23$, we have $M / N \cong L_{2}(7)$ or $L_{2}(8)$, which implies that 23||$N \mid$. Hence $O_{23}(N) \neq 1$ by Lemma 2 , which implies that $\mathrm{O}_{23}(N)=\mathrm{O}_{23}(G) \neq 1$, a contradiction.

By the same arguments as the proof of $K / H \cong A_{5}$, we show that $K / H$ cannot be isomorphic to one of the simple groups: $L_{2}(7), A_{6}, L_{2}(8), L_{2}(11), A_{7}, U_{3}(3), M_{11}, L_{3}(4), A_{8}, M_{12}$ and $M_{22}$. 
Suppose that $K / H \cong M_{23}$. Since $\left|\operatorname{Out}\left(M_{23}\right)\right|=\operatorname{Mult}\left(M_{23}\right)=1$, we have that $G \cong H \times M_{23}$, where $|H|=2^{3} \cdot 3$. By checking the character table of $M_{23}$, it is easy to see that there exists no irreducible character of degree $2^{2} \cdot 3^{2} \cdot 7 \cdot 23$ in $G$, a contradiction.

If $K / H \cong M_{24}$, since $|G|=2^{10} \cdot 3^{3} \cdot 5 \cdot 7 \cdot 11 \cdot 23$, one has that $|H|=1$ and $G=K \cong M_{24}$, which completes the proof of Theorem $B$.

Proof of Theorem C We only need to prove the sufficiency.

In this case, $|G|=2^{7} \cdot 3^{2} \cdot 5 \cdot 7 \cdot 11$ and $L(G)=385$. Let $\chi \in \operatorname{Irr}(G)$ such that $\chi(1)=L(G)=$ $5 \cdot 7 \cdot 11$. We assert that $O_{11}(G)=1$. Otherwise, we have that $\left|O_{11}(G)\right|=11$ and $O_{11}(G)$ is abelian. Hence $\chi(1)|| G / O_{11}(G) \mid$, a contradiction. Similarly, $O_{5}(G)=O_{7}(G)=1$.

If $G$ is solvable, then the Sylow 11-subgroup of $G$ is normal in $G$ by Lemma 2. But $O_{11}(G)=1$, a contradiction. Therefore, $G$ is non-solvable.

Since $G$ is non-solvable, by Lemma 1 , we get that $G$ has a normal series $1 \unlhd H \unlhd K \unlhd G$ such that $K / H$ is a direct product of isomorphic non-abelian simple groups and $|G / K| \mid$ $|\operatorname{Out}(K / H)|$. As $|G|=2^{7} \cdot 3^{2} \cdot 5 \cdot 7 \cdot 11$, we see that $K / H$ is isomorphic to one of the simple groups: $A_{5}, L_{2}(7), A_{6}, L_{2}(8), L_{2}(11), A_{7}, M_{11}, L_{3}(4), A_{8}$ and $M_{22}$.

We first assume that $K / H \cong A_{5}$. Since $\left|\operatorname{Out}\left(A_{5}\right)\right|=2$, we have $|G / K| \mid 2$ and $|H|=2^{t} \cdot 3 \cdot 7$. 11 , where $t=4$ or 5 . If $H$ is solvable, then $O_{11}(H)=O_{11}(G) \neq 1$ by Lemma 2 , a contradiction. Hence $H$ is non-solvable and $H$ has a normal series $1 \unlhd N \unlhd M \unlhd H$ such that $M / N$ is a direct product of isomorphic non-abelian simple groups and $|H / M||| \operatorname{Out}(M / N) \mid$. As $|H|=2^{t} \cdot 3 \cdot 7 \cdot 11$, one has that $M / N \cong L_{3}(2)$ and $|N|=2^{s} \cdot 11$, where $0 \leq s \leq 2$. Let $P$ be the Sylow 11-subgroup of $N$. Then $P$ is normal in $N$ by Sylow theorem. Since $P$ is also a Sylow 11-subgroup in $G$ and $N$ is subnormal in $G$, we have $P \unlhd G$, a contradiction.

Similarly, $K / H$ cannot be isomorphic to the simple groups: $L_{2}(7), A_{6}, L_{2}(8), L_{2}(11), A_{7}$, $L_{3}(4)$ or $A_{8}$.

Assume that $K / H \cong L_{2}(11)$. Since $\left|\operatorname{Out}\left(L_{2}(11)\right)\right|=2$, we have $|G / K| \mid 2$ and $|H|=2^{\alpha} \cdot 3 \cdot 7$, where $\alpha=4$ or 5 . Suppose that $H=H^{\prime}$. Then $H$ has a normal subgroup $S$ such that $H / S \cong$ $L_{2}(7)$, where $|S|=2$ or 4 . Obviously, we know that $S \leq Z(H)$, and then $S \unlhd G$. Let $\theta \in \operatorname{Irr}(S)$ such that $\left[\chi_{S}, \theta\right] \neq 0$. Then $\theta(1)=1$ since $S$ is abelian. Let $e=\left[\chi_{S}, \theta\right]$ and $t=\left|G: I_{G}(\theta)\right|$. Then $t=1$ and $e=\chi(1)=385$ by the Clifford theorem (see Theorem 6.2 in [5]). But $e^{2} \cdot t=$ $\left[\chi_{H}, \chi_{H}\right]>|G: H|$, a contradiction. Hence $H^{\prime}<H$. Suppose that $\left|H / H^{\prime}\right|=2$. Then $H / H^{\prime}$ is central in $G / H$. Let $\beta$ be an irreducible component of $\chi_{H}$, and let $\theta$ be an irreducible component of $\beta_{H^{\prime}}$. Then $\theta(1)=\beta(1)=7$ and $\theta$ is extendible to $\beta$. Hence $\lambda \beta$ is invariant in $G$ for every $\lambda \in \operatorname{Irr}\left(H / H^{\prime}\right)$ if $\beta$ is invariant in $G$. Since $|H|=2^{\alpha} \cdot 3 \cdot 7 \cdot 11$, where $\alpha=4$ or 5 , $H$ has at most 12 irreducible characters of degree 7. Let $t=\left|G: I_{G}(\beta)\right|$. Then $t \leq 12$. Since the index of the maximal subgroup of $U$ containing $I_{G}(\theta)$ divides $t$, we have that $t=1$ or 11 by checking maximal subgroups of $L_{2}(11)$ (see ATLAS table in [6]). If $t=11$, then $H$ has exactly 12 irreducible characters of degree 7, and one of them, say $\delta$, is invariant in $G$. Hence, $\lambda \delta$ is also invariant in $G$ for $\lambda \in \operatorname{Irr}\left(H / H^{\prime}\right)$, which forces $t \leq 10$, a contradiction. Therefore $t=1$ and $e=55$. But $(55)^{2}=\left[\chi_{H}, \chi_{H}\right]>|G: H|$, a contradiction. By the same reasoning as before, we can prove that $\left|H / H^{\prime}\right| \neq 2^{m} \cdot 3^{n}$, where $1 \leq m \leq 3$ and $0 \leq n \leq 1$. If $\left|H / H^{\prime}\right|=2^{m} \cdot 3^{n}$, where $m=4$ or 5 , then the Sylow 7 subgroup of $H^{\prime}$ is normal in $H^{\prime}$, and so it is normal in $G$, a contradiction. Now we assume that 7||$H / H^{\prime} \mid$. Let $H^{\prime} \leq A \unlhd H$ such that $|H / A|=7$, then $H / A \leq Z(G / A)$. Since $\operatorname{Mult}\left(L_{2}(11)\right)=2$, we have $G / A \cong H / A \times L_{2}(11)$. Hence $G$ has a normal series $1 \unlhd N \unlhd M \unlhd G$ such that $M / N \cong L_{2}(11)$ and $|N|=2^{\alpha} \cdot 3$, where $\alpha=4$ or 5 . Let $\varphi$ be an irreducible component of $\chi_{M}$, and let $\eta$ be an irreducible 
component of $\varphi_{N}$. Then $\varphi(1)=55$ and $\eta(1)=1$ by the Clifford theorem (see Theorem 6.2 in [5]). Since $|\operatorname{Aut}(N)|$ is not divided by 5 and 11 , one has that $t=\left|M: I_{M}(\eta)\right|=1$. Therefore $(55)^{2}=\left[\varphi_{N}, \varphi_{N}\right]>|M: N|$, a contradiction.

Suppose that $K / H \cong M_{11}$. Since $\left|\operatorname{Out}\left(M_{11}\right)\right|=\operatorname{Mult}\left(M_{11}\right)=1$, we have $G \cong H \times M_{11}$, where $|H|=2^{3}$. 7. Let $\theta \in \operatorname{Irr}(H)$ such that $\left[\chi_{H}, \theta\right] \neq 0$. Since $\theta(1) \mid \chi(1)$ and $\theta(1)|| H \mid$, one has that $\theta(1)=7$, which implies that $H$ is a Frobenius group with an elementary kernel of order 8 and a cyclic complement of order 7 .

Suppose that $K / H \cong M_{22}$. Since $|G|=2^{7} \cdot 3^{2} \cdot 5 \cdot 7 \cdot 11$, we have that $|H|=1$ and $G=K \cong$ $M_{22}$, which completes the proof of Theorem C.

Competing interests

The authors declare that they have no competing interests.

Authors' contributions

HX carried out the study of the Mathieu groups $M_{11}, M_{12}$ and $M_{23}$. YY carried out the study of the Mathieu group $M_{24}$. GC carried out the study of the Mathieu group $M_{22}$. All authors read and approved the final manuscript.

\section{Author details}

${ }^{1}$ School of Mathematics and Statistics, Southwest University, Chongqing, 400715, China. ${ }^{2}$ Department of Mathematics and Information Engineering, Chongqing University of Education, Chongqing, 400067, China.

\section{Acknowledgements}

Article is supported by the Natural Science Foundation of China (Grant No. 11271301; 11171364), The Fundamental Research Funds for the Central Universities (Grant No. XDJK2009C074), Graduate-Innovation Funds of Science of SWU (Grant No. ky2009013) and Natural Science Foundation Project of CQ CSTC (Grant No. cstc201 1jjA00020).

\section{Received: 29 December 2012 Accepted: 15 April 2013 Published: 26 April 2013}

\section{References}

1. Huppert, B: Some simple groups which are determined by the set of their character degrees, I. III. J. Math. 44(4), 828-842 (2000)

2. Huppert, B: Some simple groups which are determined by the set of their character degrees, II. Rend. Semin. Mat. Univ. Padova 115, 1-13 (2006)

3. Wakefield, TP: Verifying Huppert's conjecture for $P S L_{3}(q)$ and $P S U_{3}\left(q^{2}\right)$. Commun. Algebra 37, 2887-2906 (2009)

4. Tong-Viet, HP, Wakefield, TP: On Huppert's conjecture for $G^{2}(q), q \geq 7$. J. Pure Appl. Algebra 216, 2720-2729 (2012)

5. Isaacs, IM: Character Theory of Finite Groups. Academic Press, New York (1976)

6. Conway, JH, Nortion, SP, Parker, RA, Wilson, RA: Atlas of Finite Groups. Oxford University Press, Oxford (1985)

doi:10.1186/1029-242X-2013-209

Cite this article as: Xu et al.: A new characterization of Mathieu-groups by the order and one irreducible character degree. Journal of Inequalities and Applications 2013 2013:209.

\section{Submit your manuscript to a SpringerOpen ${ }^{\circ}$ journal and benefit from:}

- Convenient online submission

Rigorous peer review

- Immediate publication on acceptance

- Open access: articles freely available online

- High visibility within the field

- Retaining the copyright to your article 